\title{
Mental rotation and visual familiarity
}

\author{
ASHER KORIAT and JOEL NORMAN \\ University of Haifa, Haifa, Israel
}

\begin{abstract}
Mental rotation functions often evidence a curvilinear trend indicating relative indifference to small departures from the upright. In Experiment 1, this was true only for normal letters whereas reflected letters yielded a largely linear rotation function. This suggested that the internal representation of familiar visual patterns is characterized by broad orientation tuning that allows recognition despite small disorientations. Since familiar stimuli are often encountered slightly tilted from the upright, broad orientation tuning may reflect this ecological distribution. Experiment 2, however, indicated the possible involvement of two additional processes. Subjects were first trained on unfamiliar nonsense characters that appeared only in their "upright" positions. This was followed by a normal-reflected mental rotation task on these characters. Initially, rotation functions were more curvilinear for normal than for reflected characters. This suggested that practice with upright stimuli automatically contributes to broad tuning. Further practice resulted in a curvilinear trend for reflected characters as well, despite the fact that they appeared with equal probability in all orientations. This suggested that the very process of mentally rotating stimuli to the upright orientation increases insensitivity to slight departures from this orientation. Experiment 3 established that the different functions found for normal and reflected characters were due to stimulus rather than response factors.
\end{abstract}

The mental rotation task has been studied extensively in recent years. Ever since it was introduced by Shepard and Metzler (1971), it has proved to be fruitful in the analysis of how visual information is represented and manipulated by the cognitive system.

The most consistently obtained result with this task is the monotonic relationship between response time and the extent of mental rotation presumably required. Yet, there have been some disagreements regarding the exact shape of this function. Shepard and Metzler (1971), using pairs of three-dimensional drawings, found that the time to make "same" judgments increased with the angular deviation between the two objects in a remarkably linear manner. Cooper and Shepard (1973), on the other hand, obtained functions which clearly departed from linearity. They used alphanumeric characters in different orientations and had subjects judge whether they were normal or reflected mirror images. In the latter task, the increase in response time with angular deviation from upright indicated a quadratic trend, with small deviations yielding relatively small effects. Cooper and Shepard offered several explanations for this nonlinearity, among them that when the stimulus in question is familiar, mental rotation may not be required for small deviations from upright. This may explain why the normal-reflected single-letter task yields a nonlinear function whereas the threedimensional block task yields linear functions.

Another interpretation for the nonlinearity effect was proposed by Hock and Tromley (1978). When a visual

We are indebted to Zvia Kaplan and to Hagit Tal for programming the experiments, and to Ofra Ziv and Ilana Zoellner for running them. Requests for reprints should be sent to Asher Koriat or Joel Norman, Department of Psychology, University of Haifa, Haifa, Israel. stimulus is slightly tilted it may still be perceptually upright as long as its perceptual "top" and "bottom" remain consistent with the "top" and "bottom" of its memory representation. Different letters of the alphabet are assumed to differ in the range of orientations over which they are perceived to be upright. Within this range, no rotation is necessary for recognition.

The research reported in the present paper was based on a somewhat different account of the nonlinearity effect and the conditions under which it is obtained. It is proposed that extensive practice with a visual stimulus results in a memory representation that is broadly tuned, thus enabling efficient stimulus recognition over a relatively wide range of orientations. Small deviations from normal orientation do not require rotation before recognition. This broad tuning is due to extensive practice and occurs whatever the shape of the particular stimulus. An identical stimulus, when unfamiliar, should evidence sensitivity to misorientation and should require rotation even for small degrees of deviation from upright.

If the crucial element lies in the visual familiarity of the stimulus, then one simple test of this hypothesis is provided by a comparison of the rotation functions of normal and reflected letters. A normal letter and its reflected image share many common features, and subjects can readily identify the letter whichever format (normal or reflected) is employed (see, e.g., White, 1980). Nevertheless, subjects undoubtedly have far more practice with the normal format than with the reflected format. We would therefore expect a quadratic rotation function for normal letters and a more nearly linear function for reflected letters. This assumes that it is the familiarity of the exact visual pattern that is of importance. This implies that the difference in the shapes of the rotation functions found 
between the normal-reflected task and the threedimensional block task is due solely to the results for the normal letters; reflected letters should yield linear functions similar to those found for other nonfamiliar figures.

\section{EXPERIMENT 1}

The first experiment was run as part of a general project on the question of what is rotated in mental rotation. The exact procedure is reported elsewhere (Koriat \& Norman, 1984, Experiment 1), and it will be described only briefly here.

\section{Method}

Stimuli and Procedure. The experiment was controlled by a PDP-11/34 minicomputer. Subjects were seated with their heads resting on a chin- and headrest (to prevent head rotations) at a viewing distance of $80 \mathrm{~cm}$ from a VT11 graphic display unit. Four Hebrew letters and their mirror images served as the stimuli. Each could appear in any of the following six orientations: $0^{\circ}, 60^{\circ}, 120^{\circ}$, $180^{\circ}, 240^{\circ}$, and $300^{\circ}$. On each trial, a single letter was presented at the center of the screen and remained on until the subject responded by pressing a key with the right ("normal") or the left ("reflected") index finger. The response-stimulus interval was $500 \mathrm{msec}$.

The subjects participated in one session, which consisted of 40 practice trials followed by four blocks of 150 trials each with a short rest period between them. Each block consisted of six warm-up trials, followed by 144 experimental trials, the latter representing an equal number of each of the orientation $x$ format (normal vs. reflected) conditions. Each letter appeared equally often in each of these conditions across all four blocks. The subjects were instructed to respond as quickly as they could without making errors.

Subjects. Sixteen students, whose primary language was Hebrew, participated in the study.

\section{Results}

The following analyses were based on 576 experimental trials per subject. About $0.3 \%$ of all response times were outside the range of 250 to $5,000 \mathrm{msec}$, and were eliminated from the analyses.

Figure 1 presents mean response time for correct responses and percent errors for normal and reflected letters as a function of stimulus orientation. A two-way orientation $\times$ format analysis of variance (ANOVA) yielded significant effects for orientation $[F(5,75)=$ $70.94, \mathrm{p}<.0001]$, for format $[\mathrm{F}(1,15)=66.66, \mathrm{p}<$ $.0001]$, and for the interaction $[F(5,75)=5.50$, p $<.0005]$.

Three differences between the response time functions for normal and reflected letters are readily apparent in Figure 1, and may account for the orientation $\times$ format interaction. In comparison with reflected letters, the rotation functions for normal letters seem to evidence (1) stronger nonlinearity, (2) stronger effects of rotation, and (3) a certain degree of asymmetry.

The response-time rotation function is apparently linear for reflected letters, but seems to evidence a curvilinear trend for normal letters. The following analyses of linearity were based on the four angular deviations from the

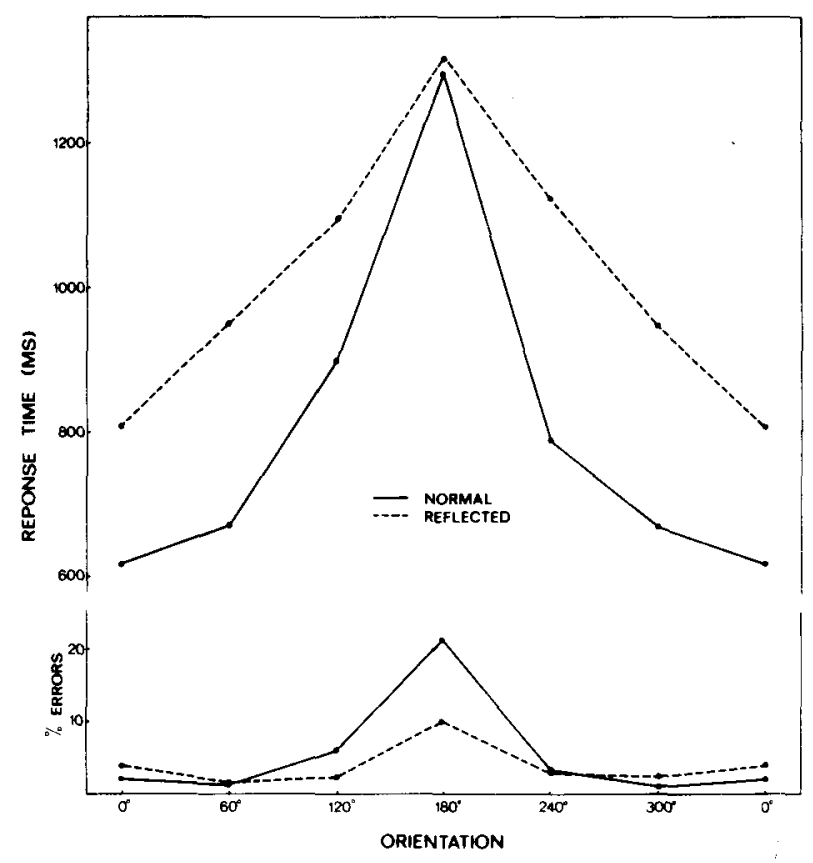

Figure 1. Mean response time (in milliseconds) and percentage of errors for normal and reflected letters as a function of orientation (Experiment 1).

upright $\left(0^{\circ}, 60^{\circ}, 120^{\circ}\right.$, and $\left.180^{\circ}\right)$, regardless of direction. For normal letters, the effects of angular deviation indicated significant linear $[F(1,45)=136.86, p<.001]$ and quadratic $[F(1,45)=22.74, p<.001]$ trends. The linear trend accounted for $85.4 \%$ of the variance, and the quadratic trend for $14.2 \%$. On the other hand, analyses on reflected letters yielded a significant linear trend $[\mathrm{F}(1,45)=110.35, \mathrm{p}<.001]$ which practically accounted for all of the variance $(99.2 \%)$. The quadratic trend accounted for only $0.7 \%$ of the variance and was not significant $(F<1)$. For normal letters, rotation rate was $1,221 \mathrm{deg} / \mathrm{sec}$ for the $0^{\circ}$ to $60^{\circ}$ range and $191 \mathrm{deg} / \mathrm{sec}$ for the $60^{\circ}$ to $180^{\circ}$ range. The respective figures for reflected letters were 424 and $329 \mathrm{deg} / \mathrm{sec}$.

If these results are taken to indicate that familiar visual shapes are broadly tuned with respect to deviations from the upright, perhaps the rotation function for the unfamiliar, reflected letters undergoes systematic changes in the course of the experiment as a result of practice. To examine this possibility, the results for the four blocks of the experiment were compared. Although there was a slight trend suggesting that the differences in the rotation curves of normal and reflected letters diminish with increased practice, a three-way orientation $x$ format $x$ block ANOVA on response time yielded a nonsignificant effect for the interaction $[F(15,224)=1.44, p<.15]$.

Let us turn now to the other two differences noted between normal and reflected letters (Figure 1). First, the overall effects of rotation are stronger for normal than for reflected letters. The extent of the rotation effects, from $0^{\circ}$ to $180^{\circ}$, was $677 \mathrm{msec}$ for normal letters and 
only $507 \mathrm{msec}$ for reflected letters. The advantage of normal over reflected letters decreases gradually with increasing angular deviation from upright, so that, for upsidedown orientations $\left(180^{\circ}\right)$, there appears to be no difference between them. Furthermore, the error data (lower panel of Figure 1) indicate that this is not due to a speedaccuracy tradeoff. Rather surprisingly, the normal letters yielded twice as many errors as the reflected letters for the $180^{\circ}$ orientation.

Second, note the asymmetry in the responses to normal letters evidenced in the comparison between the $120^{\circ}$ and $240^{\circ}$ orientations (Figure 1). A similar asymmetry was obtained for the recognition of rotated Hebrew words (Koriat \& Norman, 1985, in press). Interestingly, the results of the present study indicate that this asymmetry is entirely confined to the normal format, where responses were $115 \mathrm{msec}$ slower to the $120^{\circ}$ orientation than to the $240^{\circ}$ orientation. A two-way orientation $\left(120^{\circ}\right.$ vs. $\left.240^{\circ}\right)$ $X$ format ANOVA yielded $F(1,15)=20.49, p<.0005$, for the interaction. When only normal letters are considered, the difference between response times to $120^{\circ}$ and $240^{\circ}$ orientations yielded $\mathrm{t}(15)=4.05, \mathrm{p}<.002$, whereas that for reflected letters yielded $t(15)=1.22$, n.s. This pattern of asymmetry for normal but not for reflected letters was found to hold for each of the four letters. For the normal format, the differences between the $240^{\circ}$ and $120^{\circ}$ orientations ranged between 99 and $135 \mathrm{msec}$, and were significant for three letters $(\mathrm{p}<.01)$ and borderline for the fourth $(\mathrm{p}<.06)$.

\section{Discussion}

The results of Experiment 1 indicated significant differences in the shape of the rotation functions for normal and reflected letters. For reflected letters, response time increased in a remarkably linear manner with angular deviation from upright. Normal letters, on the other hand, exhibited a significant quadratic trend. This is consistent with the hypothesis that overlearned visual stimuli achieve a certain degree of indifference to disorientation, and their recognition is not impaired by small departures from the upright.

The nonlinearity effect may indicate that normal letters are identified even when not fully upright. Apparently, in a mental-rotation reflection task, subjects first determine the identity and orientation of a stimulus character and then mentally rotate it to the upright (see Cooper $\&$ Shepard, 1973). When the character is normal and only slightly disoriented, it may directly activate its corresponding visual code, resulting in a "normal" response. A reflected character at the same disorientation, on the other hand, might require mental rotation before a response can be made. It is still somewhat surprising, however, that the normal letters did not show an advantage over reflected letters at the $180^{\circ}$ orientation. This would have been expected if they had been recognized before being fully rotated to the objective upright. But the possibility exists that the process of direct identification of tilted normal letters holds only when the visual stimulus itself is within this range of indifference to disorientation but does not hold for a generated image of that orientation.

Some similarity exists between our results and those reported by Carpenter and Eisenberg (1978) in their study of haptic mental rotation in blind and sighted subjects. In that study, a visual mental-rotation task, which was included as a control condition, indicated more curvilinear functions for normal than for reflected letters. This curvilinear trend was also obtained in the haptic condition, but only for sighted subjects. In interpreting these results, Carpenter and Eisenberg proposed, first, that the curvilinearity effect was due to the familiarity of the visual letters and, second, that this effect extended to the haptic condition for sighted subjects because they translated the haptic stimulus into a visual representation and then mentally rotated this representation. Blind subjects, who are less familiar with letters, do not evidence such curvilinearity.

The results of Experiment 1 are consistent with the interpretation that relates the nonlinearity effect specifically to the familiarity of the visual stimulus. A reflected character typically shares several of its features with its own normal version, and may be almost as efficiently identified as a normal character (e.g., see Corballis \& Nagourney, 1978; Corballis, Zbrodoff, Shetzer, \& Butler, 1978). Yet the finding that reflected letters display the same type of rotation function as do nonsense, unfamiliar figures (e.g., Shepard \& Metzler, 1971) suggests that the nonlinearity effect depends on the availability of an overlearned code for the visual pattern as a whole rather than on the availability of an abstract, nominal code.

The results of Experiment 1 do not support one interpretation of the nonlinearity effect considered by Cooper and Shepard (1973; see also Kosslyn, 1980), namely, that it reflects "acceleration" of the rate of mental rotation as initial "inertia" is overcome. If this were the case, nonlinearity should have been found for reflected characters as well.

The difference in slope between normal and reflected letters is consistent with results obtained for tasks that required the matching of two nonsense figures that differ in orientation (e.g., Carter, Pazak, \& Kail, 1983; Pellegrino \& Kail, 1982). Those results indicated steeper rotation slopes for "same" than for "different" judgments, and were taken to suggest that "different" responses were based on a more extensive comparison than "same" responses. It may be speculated that a similar process occurs when a visual stimulus is matched against an internal representation as when it is matched against a second visual stimulus. We should therefore expect the functions to be flatter for "different" than for "same" responses and flatter for "reflected" than for "normal" alphabetic stimuli.

The asymmetry effect found for normal but not for reflected letters is not apparent in the curves of Cooper and Shepard (1973), perhaps because they combined data for normal and reflected letters. However, a pattern of asymmetry very similar to ours may be noted in the figures 
of Carpenter and Eisenberg (1978; Figures 2 and 3, p. 120), which indicate longer response times for the $120^{\circ}$ than for the $240^{\circ}$ orientation, but only for normal letters. More recently, Robertson and Palmer (1983), using large letters made up of small letters, found longer response times for $120^{\circ}$ than for $240^{\circ}$ for normal large letters and the reverse for reflected letters. Also, the same type of asymmetry found in the present study for normal letters was also obtained in lexical decision tasks using rotated Hebrew strings (Koriat \& Norman, 1985, in press).

Robertson and Palmer suggested that the asymmetry might be due to the possibility that the direction in which the letter faces interferes with rotating the figure in the opposite (shortest) direction. However, the observation that the asymmetry pattern was the same for both Hebrew (which is a left-going script) and English is inconsistent with this interpretation.

Asymmetry in mental rotation has been reported for a variety of stimuli (Chou, 1929; Dearborn, 1899; Simion, Bagnara, Roncato, \& Umiltà, 1982; Smith, Cambria, \& Stefan, 1964), but these effects are quite difficult to interpret. The finding that the asymmetry is confined to normal letters might help identify the source of this effect. We shall return to this issue in connection with Experiment 2 .

\section{EXPERIMENT 2}

The nonlinearity effect obtained in Experiment 1 was interpreted as indicating that the internal representation of familiar visual stimuli was broadly tuned. The major aim of Experiment 2 was to obtain information regarding the possible origin of the relative insensitivity to small departures from the upright. We propose three hypotheses regarding the mechanism underlying broad orientation tuning: the "ecological distribution" hypothesis, the "automatic broadening" hypothesis, and the "mental rotation"' hypothesis. Each of these hypotheses implies a different mechanism. These mechanisms are not mutually exclusive, and one or more of them may contribute to the observed effects.

The ecological distribution hypothesis is probably that implied in previous explanations of the nonlinearity effect in terms of stimulus familiarity. It claims that the broad tuning simply mimics the ecological distribution of stimulus orientations. Although familiar stimuli tend to have a standard "upright" orientation, they are rarely encountered in this exact orientation. The distribution of orientations in our perceptual environment tends to be clustered about the upright. This, of course, may result in visual codes with broad orientation tuning, and may explain the results of Experiment 1. That broad orientation tuning might be achieved by extensive training on different orientations of a visual stimulus has been demonstrated by Shinar and Owen (1973). Their subjects first memorized visual stimuli at one orientation, and then had to decide whether stimuli appearing at disorientations of up to $90^{\circ}$ matched or did not match the stimulus. Although during the first session "match" reaction times increased with degree of rotation, this effect disappeared entirely with practice, suggesting that subjects were responding to some information that was insensitive to orientation. Thus, practice in classifying stimuli that appear in different orientations may result in broad orientation tuning, and the orientation tuning of a memory representation may simply reflect the distribution of the stimulus orientations in the perceptual environment.

The automatic broadening hypothesis claims that broad orientation tuning is an intrinsic by-product of extensive practice, and is acquired for well-learned stimuli even when these are always encountered at the same "upright" orientation. Practice with a visual pattern increases the plasticity of its memory representation, enabling it to be activated by an increasingly large range of variants of the original pattern. Thus, broad orientation tuning would be seen as an intrinsic correlate of automaticity.

The mental rotation hypothesis claims that broad orientation tuning results from processes of mental rotation. When a misoriented familiar stimulus is encountered, it tends to induce mental rotation intended to rectify it. If the disoriented stimulus is imagined to rotate through all intermediate orientations, then, in imagery, small disorientations from the upright should be experienced more often than larger ones. This distribution of imagined orientations about the upright may be the basis for the broad tuning of familiar stimuli. If broad tuning mimics the distribution of imagined rather than perceived stimulus orientations, then repeated exposure to a disoriented stimulus should result in a range of indifference around the upright orientation, even if the stimulus occurs equally often at all orientations in the external environment. Thus, according to this hypothesis, mental rotation is the vehicle by which internal representations acquire their broad orientation tuning.

Experiment 2 focused on the automatic broadening and mental rotation hypotheses. To evaluate these two hypotheses, one must be able to control the ecological distribution of the various stimulus orientations. Therefore, unfamiliar nonsense characters were employed. Half of the subjects were trained on one set of four nonsense characters, and the other half were trained on their reflected images. In the training phase, each of the characters appeared only in one ("upright") orientation. Both groups were then presented with a mental rotation task and required to decide whether the character was in its "normal" (i.e., trained) format or in a "reflected" format. In this phase, the characters occurred equally often in each of six orientations. If the "automatic broadening" hypothesis was correct, then the rotation curves for "normal" characters should evidence significant nonlinear effects, whereas those for reflected characters should be largely linear during the earliest phase of the mental rotation task, despite the fact that the normal characters were experienced in only one orientation during training. If the "mental rotation" hypothesis was correct, then the reflected characters should evidence increasing cur- 
vilinearity with increasing practice, despite the fact that they were encountered equally often in all orientations during the mental rotation phase of the experiment.

An ancillary aim of Experiment 2 was to explore the origin of the asymmetry effect found in Experiment 1. For three of the letters used in that experiment (and probably for the fourth as well), the critical feature for distinguishing the letter from its reflected image was on the left side of the letter. In Experiment 2, the side of the "landmark feature"' (see Hochberg \& Gelman, 1977) was manipulated. Two characters had their landmark features on the left side and two on the right side. Since half of the subjects were trained on one version of each character while the other half were trained on its reflected image, it was possible to study the effects of landmark side both within groups and between groups. We examined the possibility that (1) the asymmetry in the rotation function is found only for "normal" (trained) characters, and (2) for these characters, the pattern of asymmetry varies systematically with the side of the landmark feature. If these predictions are supported, they may narrow down the possible causes of the asymmetry effects.

\section{Method}

Stimuli. Four characters were constructed to bear little similarity to Latin or Hebrew letters. These are presented in their "upright" orientations in Figure 2. In this orientation, all four characters appeared to be perceptually stable. They appeared in two versions, which were mirror images of each other. For each version, two characters (labeled A and B) had a distinctive feature on one side and the other two (labeled C and D) had the landmark feature on the other side. When the characters appeared on the graphic

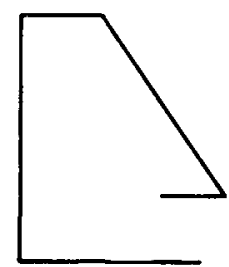

(A)

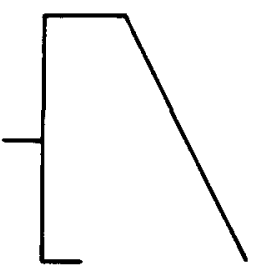

(C)

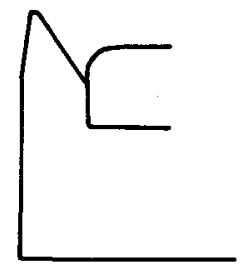

(B)

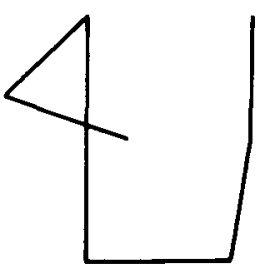

(D)
Figure 2. The four characters used in Experiment 2 in their "upright" orientation in one version. (In the second version, mirror images of these characters were used.) display unit, they subtended about $1.8 \mathrm{~cm}$ horizontally and vertically.

Design. Subjects participated in three sessions on 3 separate days, with the restriction that the entire experiment had to be completed within 4 days. The first two sessions consisted of training in the identification of the four characters. The subjects were presented with only one version of each character and were required to respond to each character by pressing one of four designated keys, using the index and middle fingers of the two hands. Half of the subjects were trained with the first version, and half with the second version. The third session was divided into two parts, both involving mental rotation. Before the mental-rotation task was introduced, two brief preliminary tasks were administered. The first was a brief repetition of the training task (Sessions 1 and 2), and the second required that the subjects distinguish "normal" from "reflected" characters, with all the characters presented only in the upright $\left(0^{\circ}\right)$ orientation.

The mental rotation task was then administered. Each group of four subjects received the same procedure in the training and mental rotation tasks, except that in the training phase two subjects were presented with the first version of the characters and two were presented with the second version. The assignment of particular response keys to particular characters was the same for each group of four subjects but differed among the four groups. For all groups, however, the two characters with landmark features on the same side were each assigned to a different hand. In addition, of the pair of subjects receiving the same training version, one was required to respond "normal" with the right hand and the other to do so with the left hand.

Procedure. The apparatus was the same as that used in Experiment 1 . In the first session, the subjects were told that the study dealt with the question of how quickly people can learn to respond automatically to letters they have not seen before. They were informed that they would see four unfamiliar "letters," one at a time, and that they would have to respond to each by pressing one of four keys using the middle and index fingers of the two hands. The subjects sat at a viewing distance of $80 \mathrm{~cm}$, with their heads resting on a chin- and headrest, which prevented head tilt. A series of 40 practice trials was then presented, and the subjects were asked to discover, through trial and error, which key corresponded to which character. On each trial, the character was presented until the subject pressed the correct key. If the key pressed first was incorrect, this was counted as an error; if it was correct, the response time was recorded. There was a 500 -msec response-stimulus interval between trials.

There were four experimental blocks of 150 trials each, in which the characters appeared at random. At the end of the practice block and of each experimental block, the subjects were informed of their mean response times for correct responses and percent errors for that block and required to write them down. They were encouraged to improve their performance from one block to the next. After each block, the subjects were asked to try to draw the four characters to the best of their ability.

At the completion of the fourth block, the subjects were given a 5-min break, which was followed by a repetition of the full procedure of one practice block and four experimental blocks.

The second session was an exact replication of the first session. The third session begar with one practice block of the training phase, to refresh the subjects' memory. Immediately afterwards, the subjects were shown the four upright characters in either their "normal" or their "reflected" formats, and required to decide whether each character was normal or reflected. There were $\mathbf{8 0}$ trials, and the subjects received verbal feedback after each. The mental rotation task followed. The subjects were told that they would see the four characters in one of six orientations and that they should indicate, as quickly as they could without making errors, whether the character was "normal" (i.e., in the trained format) or reflected. The details of the procedure were exactly those of Experiment 1, with the following exceptions: (1) The subjects were given verbal 
feedback ("right" -“"wrong") after each of the 40 practice trials; (2) half of the subjects responded to "normal" with the right index finger and half with the left index finger; (3) when the subjects completed the four blocks (Part 1), they were asked to take a 5-10-min break prior to commencing Part 2, in which the entire mental rotation procedure of one practice block and four experimental blocks was repeated. Thus, altogether, the mental rotation task consisted of eight blocks of 144 experimental trials each.

Subjects. Sixteen paid subjects participated in the study. Four additional subjects with $15 \%$ errors or more in the mental rotation phase were replaced by four new subjects.

\section{Results}

We shall first examine the results of the training phase. In the analyses of this phase, response times outside the 200 - to 2,500 -msec range were not included $(4.6 \%)$. Mean response times in each of the 16 blocks of this phase indicated a steady decline over the 8 blocks in the first session, and superior, apparently asymptotic performance in the 8 blocks of the second session. Breaking these data down by groups of 4 blocks each, 2 in each session, the respective means were $752,670,633$, and $629 \mathrm{msec}$. In other words, there was evidence of overlearning in the second session of this phase. The error data indicated an even quicker drop to asymptotic performance, with the respective error percentages for the four groups of four blocks yielding $5.95 \%, 3.28 \%, 2.97 \%$, and $3.32 \%$ errors.

In the analyses of the mental rotation phase, response times outside the 250 - to 6,000 -msec range were not included $(0.3 \%$ of all responses). Figure 3 presents mean

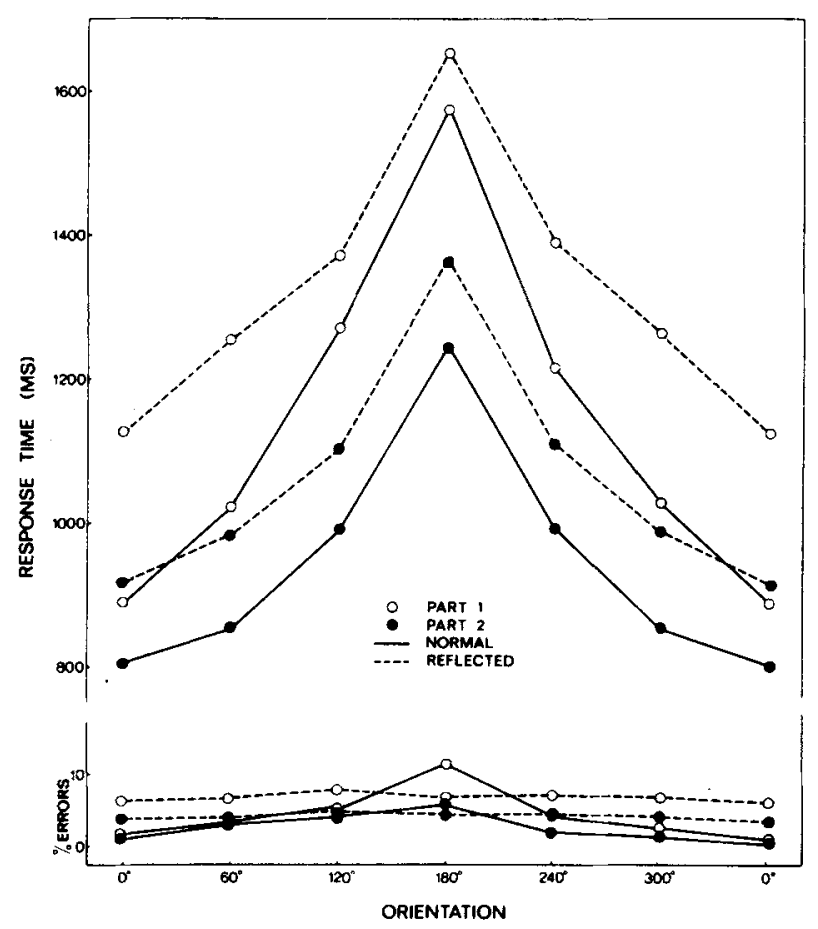

Figure 3. Mean response time (in milliseconds) and percentage of errors for "normal" and "reflected" characters in Parts 1 and 2 as a function of orientation (Experiment 2). response time and percent errors for "normal" and "reflected" characters for Parts 1 and 2 as a function of orientation. A preliminary three-way ANOVA on these data indicated significant effects for format $[F(1,15)=$ $40.59, \mathrm{p}<.0001]$, for orientation $[F(5,75)=37.89$, $<.0001]$, and for part $[\mathrm{F}(1,15)=21.76, \mathrm{p}<.0001]$. The orientation $\times$ part interaction was significant $[F(5,75)=2.66, p<.05]$, as was the triple interaction $[\mathrm{F}(5,75)=3.08, \mathrm{p}<.025]$.

The results for Part 1 are pertinent to the automatic broadening hypothesis. According to this hypothesis, the nonlinearity effect should be found for normal characters but not for reflected characters. A two-way orientation $X$ format ANOVA for this part yielded highly significant main effects for both factors, and the interaction was also significant $[\mathrm{F}(5,75)=3.08, \mathrm{p}<.025]$. Analyses of linearity were carried out, using deviations from the upright. For normal characters, these were significant for both the linear $[F(1,45)=119.49, p<.0001]$ and the quadratic $[F(1,45)=4.52, p<.05]$ trends. The linear trend accounted for $96.3 \%$ of the variance, and the quadratic trend for $3.7 \%$. For reflected characters, these analyses yielded a significant linear trend $[F(1,45)=69.33$, $\mathrm{p}<.0001$ ], which accounted for $96.2 \%$ of the variance. The quadratic trend accounted for $2.9 \%$ of the variance and was not significant $[F(1,45)=2.08]$.

Thus, the results indicate a slight quadratic trend for normal characters but not for reflected ones. This is consistent with the automatic broadening hypothesis, that repeated exposure to a visual stimulus in a fixed orientation may result in the establishment of an internal representation with broad orientation tuning.

The mental rotation hypothesis predicts that practice in mental rotation should result in increased quadratic trends in the rotation functions of both normal and reflected characters. It may be seen (Figure 3) that the difference in the shape of the rotation curves for normal and reflected characters obtained in Part 1 disappears in Part 2. Thus, an orientation $\times$ format ANOVA for the second part alone indicated a nonsignificant interaction $(F<1)$. For this part, angular deviations of both normal and reflected characters yielded significant quadratic trends. These trends accounted for $9.0 \%$ of the variance for normal characters $[F(1,45)=11.76, p<.01]$ and for $7.8 \%$ of the variance for reflected characters $[F(1,45)$ $=7.40, \mathrm{p}<.01]$.

The major change from Part 1 to Part 2 occurs in response to reflected letters at upright or near-upright orientations. A two-way format $\times$ part ANOVA using only angular deviations of $120^{\circ}$ and $180^{\circ}$ yielded a nonsignificant interaction $(F<1)$, whereas the same analysis carried out for angular deviations of $0^{\circ}$ and $60^{\circ}$ yielded $F(1,15)=17.08$, $p<.001$, for the interaction.

On the basis of these results, it appears that the rotation function for both normal and reflected characters evidence a systematic increase in the size of the quadratic component with practice. In order to trace these practice effects in greater detail, the data of the eight blocks of 
the two parts were combined to form four pairs, each pair based on 288 trials. The percentage of variance accounted for by the quadratic component increased systematically for the reflected characters, $1.4 \%, 3.7 \%, 6.2 \%$, and $9.2 \%$ for the four block-pairs, respectively. The respective percentages for the normal characters were $3.3 \%, 3.9 \%$, $3.2 \%$, and $16.8 \%$.

The results lend some support to both the automatic broadening and the mental rotation hypotheses. First, the results for the familiar, normal characters yielded a slight indication that exposure to a visual stimulus in one orientation might also contribute to the establishment of a broadly tuned internal representation. Second, the rotation function for reflected characters changed systematically with practice. Although, in the first block-pair, it was by and large linear, it evidenced a significant quadratic component by the third block-pair. This change is of particular interest in view of the fact that, during the mental-rotation task, the reflected characters appeared equally often in each of the six orientations. Thus, repeated exposure to a nonfamiliar stimulus that appears in different degrees of rotation from its " upright" orientation results in increased insensitivity to small deviations from this orientation.

Let us now turn to the ancillary issue of the asymmetry in the rotation function. It may be seen in Figure 3 that the asymmetry in responding to the $120^{\circ}$ and $240^{\circ}$ orientations is found only for normal characters in Part 1. This difference is considerably smaller than that obtained in Experiment 1 (Figure 1) and is not quite significant [t $(15)$ $=2.11, \mathrm{p}<.10$, two-tailed]. It should be recalled that two of the four characters had their distinctive features on the right side and two had them on the left, and that they were reversed for half the subjects. Examination of the results for individual characters did not yield any systematic relationship between asymmetry and side of the distinctive feature for normal characters. In sum, the results of Experiment 2 cannot help in delineating the source of the asymmetry effect observed in Experiment 1, and this issue must await further research.

\section{Discussion}

The results of Experiment 2 further established and qualified the relationship between the extent of the quadratic component and the familiarity of the visual stimulus. As already noted, normal alphabetic characters are usually encountered in a variety of orientations, mostly those involving small deviations from the upright. This may account in part for the broader tuning of their representation, that is, their insensitivity to small deviations from the upright. It might be argued, therefore, that rotation functions reflect nothing more than the ecological distribution of the orientations of letters or words in our visual environment. The results of Experiment 2 indicate that this is not the whole story. First, in the earlier phase of the mental rotation task, the curve for normal, previously learned characters evidenced a slight degree of curvilinearity, whereas that for the reflected, new characters was by and large linear. This occurred despite the fact that these characters had appeared only in the upright orientation during training. Second, repeated exposure to the rotated stimuli resulted in a curvilinear rotation function for the newer, reflected characters, despite the fact that they appeared in all orientations with the same probability.

The first of these findings is consistent with the "automatic broadening" hypothesis, and suggests that broad tuning might be an intrinsic characteristic of the internal representation of well-learned visual stimuli. Although the evidence in favor of this generalization is still quite weak, this idea deserves further investigation.

The "mental rotation" hypothesis is somewhat more clearly supported by the data. The increase in the quadratic effects for reflected letters suggests that this effect may be due to the requirement for mental rotation. This requirement was assumed to provide the mechanism for broadening the range of orientations over which the internal representation of a visual stimulus might be efficiently activated. If the process of mental rotation transpires in a manner similar to that described by Shepard and Cooper (1982) (in that disoriented stimuli are imagined to rotate through all intermediate orientations), then, as far as the generated images are concerned, orientations near the upright are much more frequently experienced than are the much more removed disorientations. This is true even if the distribution of the various orientations in the external world is homogeneous.

\section{EXPERIMENT 3}

Experiments 1 and 2 (Part 1) yielded different rotation functions for normal and reflected characters. These were interpreted in terms of stimulus familiarity. But these differences could result from response characteristics rather than from stimulus characteristics. Thus, it has been proposed that "different" responses are based on a more extensive comparison than "same" responses in tasks requiring the matching of two figures at different orientations (e.g., Pellegrino \& Kail, 1982). If "reflected" responses also involve more extensive checking than "normal" responses in a mental rotation task, perhaps it is this difference that is responsible for the observed differences in the nonlinearity effect.

In Experiment 3, we attempted to unconfound the contributions of stimulus characteristics (e.g., stimulus familiarity) from those of response characteristics (e.g., the requirement for "normal" or "reflected" responses). Subjects were presented with the same four Hebrew characters as in Experiment 1. However, prior to each stimulus presentation they were given advance information as to the identity of the upcoming character, and were asked to anticipate this character in a specified format. They were told to respond "same" if the character appeared in the predesignated format and "different" if it appeared in a different format. The design allowed examination of all combinations of three orthogonal factors: 
orientation, stimulus type (normal vs. reflected), and response type ("same" vs. "different").

This design allows an evaluation of three possible interpretations of the differences observed between normal and reflected characters. The first, noted above, is that these reflect differences in the processes underlying "normal" (or "same") and "reflected" (or "different") responses. If this is the case, the nonlinearity effect should obtain for "same" responses to reflected characters but not for "different" responses to normal characters.

The second interpretation is simply that since "normal" responses are generally faster than "reflected" responses, the flattening of the rotation curves about the $0^{\circ}$ orientation for "normal" responses may simply stem from a floor effect. If "same" responses in Experiment 3 are indeed found to be faster than "different" responses, we may then determine whether the nonlinearity effect still obtains for the slower "different" responses to normal characters.

The third interpretation proposes that imagined forms tend to have a less narrowly delimited orientation than their perceived counterparts. Therefore, when a visual stimulus is to be matched against an imagined stimulus, "same" responses are expected to yield nonlinear rotation functions. If this hypothesis is correct, the nonlinearity effect should be obtained even for a reflected character when it is expected by the subject. This reasoning may be extended to account for the nonlinearity effect in general. We may assume that when a mental rotation task involves a small number of characters, the representations of these characters (in their normal format) are activated in imagery, and it is this activation that is responsible for the nonlinearity effect found for normal characters.

\section{Method}

Subjects. Sixteen University of Haifa students participated in the study. Eight received course credit, and eight were paid for participating.

Apparatus and Stimuli. The apparatus was the same as that used in Experiment 1. The target stimuli consisted of the same four Hebrew letters and their mirror images used in Experiment 1. These appeared in the same six orientations as in that experiment.

Procedure. The subjects sat with their heads resting on a chinand headrest, preventing head rotations. Viewing distance was $80 \mathrm{~cm}$. At the beginning of each trial, a horizontal array of four squares appeared about $7 \mathrm{~cm}$ below the center of the screen; the squares were numbered from 1 to 4 , with the number appearing at the top of each square. The subjects were told that each square corresponded to one of the four Hebrew letters (in alphabetical order) and that they were required to learn this correspondence. They were also told that on each trial a plus or a minus sign would appear at the center of one of the squares, and that when a plus sign appeared in a particular square they were to expect the corresponding character in its normal format, whereas if a minus sign appeared they were to expect it in its reflected format.

Upon presentation of the cue, the subjects were to indicate aloud what letter and what format was expected and to press a bar when they were ready. After a 500-msec interval, the character appeared at the center of the screen. The subjects then responded by pressing one key labeled "same" or a second key labeled "different," "same" if the character appeared in the expected format and "different" if not. Half of the subjects used the right index finger for "same" and the left index finger for "different"; for the other half, the reverse pattern was employed.

The session began with 40 practice trials, followed by eight blocks of 100 trials each. Stimuli appeared at the center of the screen until the subject responded. There was a 500-msec response-stimulus interval. Each block of 100 trials consisted of four warm-up trials followed by 96 trials that represented all combinations of letter (4) $\times$ orientation $(6) \times$ expected format $(2) \times$ actual format $(2)$ in a random order.

\section{Results and Discussion}

Response times outside the range of $200-5,000 \mathrm{msec}$ were not included in the analyses $(1.1 \%)$. Figure 4 presents mean response time for correct responses as a function of orientation for different combinations of stimulus type and response type. A three-way ANOVA indicated strong main effects of orientation $[F(5,75)=44.17$, $\mathrm{p}<.0001]$, stimulus type $[\mathrm{F}(1,15)=20.15, \mathrm{p}<.0005]$, and response type $[F(1,15)=20.62, p<.0005]$. Two interactions were also significant: stimulus type $\times$ response type $[F(1,15)=67.25, p<.0001]$ and orientation $\times$ stimulus type $[F(5,75)=3.91, p<.005]$. The latter interaction (see Figure 4) stems from the response type's having a very strong effect for normal characters but a negligible effect for reflected characters. For normal characters, "same" responses averaged 1,026 msec and "different" responses averaged $1,295 \mathrm{msec}$. The respective means for reflected characters were 1,311 and 1,302 msec.

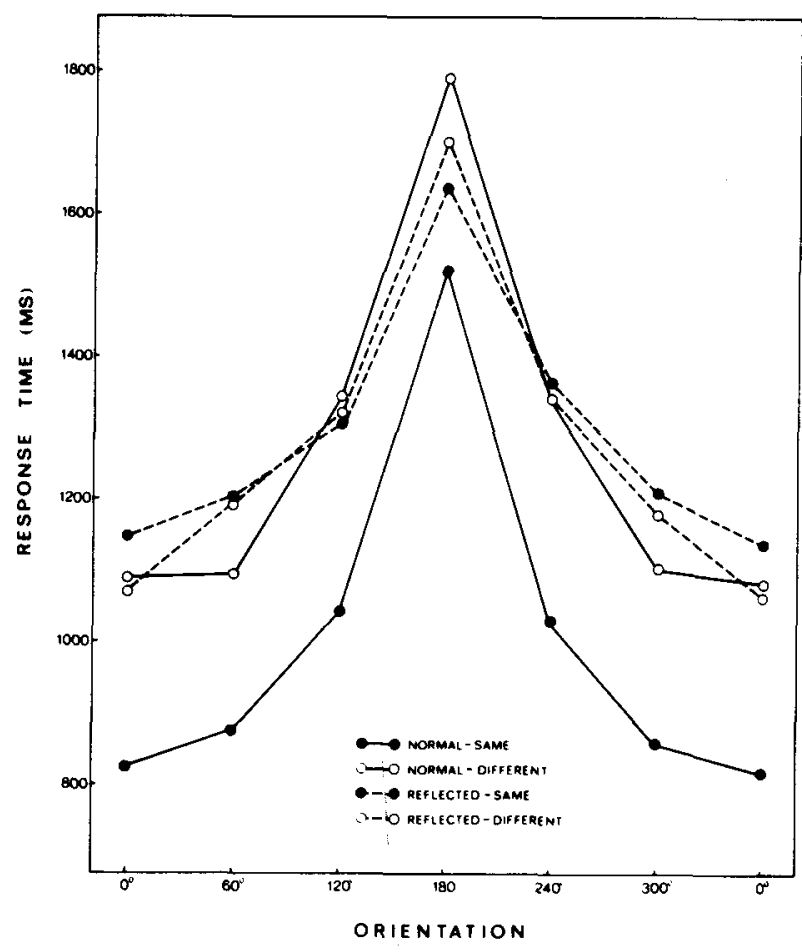

Figure 4. Mean response time (in milliseconds) as a function of orientation by stimulus type (normal vs. reflected) and response type ("same" vs. "different"). 
The orientation $\times$ stimulus type interaction, which constitutes the main focus of the present study, seems to reflect two effects. First, as in Experiment 1, the overall effect of rotation is stronger for normal than for reflected characters. The extent of the rotation effect, from $0^{\circ}$ to $180^{\circ}$ angular deviations, was $703 \mathrm{msec}$ for normal characters and $562 \mathrm{msec}$ for reflected characters. Thus, the steeper slope found for normal characters in Experiment 1 seems to be due to stimulus characteristics rather than to response characteristics. Apparently, when a reflected character is presented, subjects are not content to rotate it to an upright position, but continue to check whether there is some other transformation that might result in a normal character.

Second, it appears that the nonlinearity effect is obtained to a larger extent for normal than for reflected characters. Thus, although the differences are not as marked as those found in Experiment 1 (Figure 1), it appears clear from Figure 4 that the extent of the nonlinearity effect varies with stimulus type more than with response type. In contrast to Experiment 1, the results of the present experiment indicated significant quadratic trends for all four functions relating response time to angular deviations from the upright. However, for normal characters, these quadratic trends accounted for $15.98 \%$ of the variance for "same" responses and for $14.59 \%$ of the variance for "different" responses. The figures for reflected characters were $9.80 \%$ and $6.91 \%$, respectively.

Although "different" responses to normal characters were relatively slow, they displayed the same relative indifference to small deviations from the upright as found for the faster "same" responses to normal characters. In fact, for the former responses, the increase in response time from $0^{\circ}(1,088 \mathrm{msec})$ to $60^{\circ}(1,100 \mathrm{msec})$ deviations was slight and not significant $[t(15)=0.54]$. These results allow us to reject the possibility that the nonlinearity effect found for familiar stimuli (Figure 1) is simply due to a floor effect.

A three-way ANOVA was also carried out on percent errors. The effects of orientation generally mimicked those of response time $[F(5,75)=3.88, p<.004]$. Percent errors for "same" responses was $4.7 \%$ compared to $7.02 \%$ for " different" responses $[F(1,15)=5.34$, $\mathrm{p}<$ $.05]$. The orientation $\times$ stimulus type interaction was significant $[F(5,75)=3.16, p<.02]$ : Percent errors were slightly higher for "different" responses than for "same" responses, except for the $180^{\circ}$ orientation, which yielded $15.0 \%$ errors for "same" responses and $8.2 \%$ errors for "different" responses.

We should finally note that the asymmetry effect does not occur with the task used in Experiment 3. The reasons for this are not clear.

\section{GENERAL DISCUSSION}

The present study investigated the origin of the nonlinearity effect in rotation functions. The function relating response time to angular deviation from the upright is remarkably linear for the Shepard and Metzler (1971) task. However, it displays a marked quadratic trend in the Cooper and Shepard (1973) task, indicating relative indifference to small departures from the upright. In interpreting this discrepancy, it has been proposed that the nonlinearity effect stems from the extensive experience with alphanumeric characters (see Cooper \& Shepard, 1973; Kosslyn, 1980; Young, Palef, \& Logan, 1980). The present study yielded evidence favoring this proposition, and indicated that the nonlinearity effect was specifically related to the familiarity of the visual stimulus. It also provided information relating to the mechanism underlying this relationship.

We proposed that extensive practice with a visual stimulus results in the establishment of a broadly tuned memory representation that allows direct stimulus recognition over a relatively wide range of stimulus orientations. Consistent with this idea, the results of Experiment 1 indicated that the nonlinearity effect is entirely confined to normal letters, whereas reflected letters display a remarkably linear rotation function. The results of Experiment 3 further indicated that these differences were due to the differences between normal and reflected stimuli, and could not be accounted for by factors associated with the type of response solicited.

These results are surprising in view of the similarity between a letter and its mirror image. The finding that reflected letters yield a linear rotation function like that found for the unfamiliar three-dimensional blocks suggests that the critical factor responsible for the nonlinearity effect lies in the familiarity of the visual code. In terms of Posner's (1978) distinction, it would seem that broad tuning depends on the familiarity of the physical code rather than on that of the nominal code.

This account of the nonlinearity effect differs from that proposed by Hock and Tromley (1978), although theirs also emphasizes the importance of visual factors. They obtained quadratic effects for letters assumed to have a wide range of orientations for which they remained perceptually upright (such as $\mathrm{L}$ and $\mathrm{J}$ ), but linear effects for letters with a narrower range (such as $\mathbf{G}$ and e). Their results suggest that if a stimulus is familiar enough to have a perceptually defined "top," the shape of the rotation function depends on its visual characteristics. The present study suggests that the nonlinearity effect may not be entirely accounted for in terms of characteristics that are inherent in the visual shape of the stimulus, but depends in addition on the perceiver's past experience, that is, on the manner in which the stimulus is represented in memory.

How does extensive experience with a visual stimulus help in establishing a broadly tuned internal representation? A developmental perspective might prove instructive. If broad tuning depends on stimulus familiarity, children may be expected to yield a more linear rotation function for letters than adults. This has been found to be the case, and has been interpreted as indicating that children have a narrower range of perceptual uprightness 
(Childs \& Polich, 1979; Young et al., 1980). Obviously, mental rotation would occur only for stimuli that possess a "natural" upright orientation (see Braine, 1978). The developmental data, considered in conjunction with the present study, paradoxically suggest that these stimuli first acquire a narrowly defined perceptual upright. It is only through further experience that this definition undergoes gradual relaxation.

Assuming that the child's internal representation of alphanumeric characters is narrowly tuned, how does further experience result in a broadening of the range of perceptual uprightness? Three processes may account for this change. The first process, ecological distribution, assumes that familiar visual stimuli are normally encountered in different orientations centered around the "upright" orientation. Broad tuning simply reflects the relative distributions of the orientations in the perceptual environment. It is this mechanism that appears to have been implied in previous discussions which have attributed the nonlinearity effect to stimulus familiarity. According to this mechanism it must be assumed that the developmental trend noted above stems from adults' being exposed to a broader range of orientations of alphabetic characters.

A second process, automatic broadening, assumes that broad orientation tuning is an inherent by-product of perceptual experience. Thus, repeated exposure to a stimulus, even at a fixed orientation, should automatically contribute to increased insensitivity to small departures from this orientation. Some evidence for such a process was obtained in Experiment 2. Experience in seeing nonsense figures in one ("upright") orientation resulted in nonlinearity effects for these figures but not for their reflected images. The differences obtained were small in comparison to those found for alphabetic characters (Experiment 1). But the amount of practice allotted in Experiment 2 was also negligible compared with the experience that subjects have had with normal letters. The idea of automatic broadening deserves further exploration in view of its implications for the manner in which perceptual experience may contribute to increased plasticity of perceptual schemata. This plasticity may allow such schemata to be activated by a large range of variants of the original pattern.

The third process, mental rotation, assumes that the internal representation of a visual stimulus is initially characterized by a narrowly defined perceptual upright, and that further experience in bringing disoriented stimuli to this upright orientation helps to broaden the orientation tuning of the internal representation. When disoriented stimuli are imagined to rotate, orientations near the upright are experienced more frequently in imagery than are the more extreme disorientations. Some support for this idea comes from the finding that the rotation function for reflected characters changed systematically with practice (Experiment 2): Although it was linear by and large in the first part of the mental rotation task, it evidenced a significant quadratic component in the second part. This occurred in spite of the fact that during the mental rota- tion task the reflected characters appeared equally often in each of the six orientations.

Like the automatic broadening account, the mental rotation account may also represent a specific instance of a more general perceptual process. Objects may be assumed to have a prototypical (or canonical) form that is characterized by a specific perspective, orientation, size, etc. (see Palmer, Rosch, \& Chase, 1981). However, these objects are likely to be encountered in many different variations. We may speculate that once a prototypical visual concept has been formed to represent a particular object, further encounters with that object in many variations broaden the perceptual tuning of this concept. The process underlying this change may involve the use of internal rectifying operations that are designed to reconstruct a prototypical image from the given variant. To the extent that these rectifying operations involve continuous transformations, the generated intermediate representations may help to broaden the range of variants that may be admitted by a perceptual concept. A process of this sort may contribute to the development of abstract visual concepts (see Posner \& Keele, 1968) and perceptual constancies.

\section{REFERENCES}

Braine, L. G. (1978). A new slant on orientation perception. American Psychologist, 33, 10-22.

Carpenter, P. A., \& Eisenberg, P. (1978). Mental rotation and frame of reference for blind and sighted individuals. Perception \& Psychophysics, 23, 117-124.

Carter, P., Pazak, B., \& Kail, R. (1983). Algorithms for processing spatial information. Journal of Experimental Child Psychology, 36, 284-304.

Childs, M. K., \& Polich, J. M. (1979). Developmental differences in mental rotation. Journal of Experimental Child Psychology, 27, 339-351.

CHOU, S. K. (1929). Reading and legibility of Chinese characters. I. Journal of Experimental Psychology, 12, 156-177.

COOPER, L. A., \& ShePard, R. N. (1973). Chronometric studies of the rotation of mental images. In W. G. Chase (Ed.), Visual information processing. New York: Academic Press.

Corballis, M. C., Nagourney, B. A. (1978). Latency to categorize disoriented alphanumeric characters as letters or digits. Canadian Journal of Psychology, 32, 186-188.

Corballis, M. C., Zbrodoff, N. J., Shetzer, L. I., \& Butler, P B. (1978). Decisions about identity and orientation of rotated letters and digits. Memory \& Cognition, 6, 98-107.

Dearborn, G. V. N. (1899). Recognition under objective reversal. Psychological Review, 6, 395-400.

Hochberg, J., \& Gelman, L. (1977). The effect of landmark features on "mental rotation" times. Memory \& Cognition, 5, 23-26.

Hock, H. S., \& Tromley, C. L. (1978). Mental rotation and perceptual uprightness. Perception \& Psychophysics, 24, 529-533.

KORIAT, A., \& NORMAN, J. (1984). What is rotated in mental rotation? Journal of Experimental Psychology: Learning, Memory and Cognition, 10, 421-434.

KORIAT, A., \& Norman, J. (in press). Reading rotated words. Journal of Experimental Psychology: Human Perception and Performance.

KosslyN, S. M. (1980). Image and mind. Cambridge, MA: Harvard University Press.

Palmer, S., Rosch, E., \& Chase, P. (1981). Canonical perspective and the perception of objects. In J. B. Long \& A. D. Baddeley (Eds.), Attention and Performance IX. Hillsdale, NJ: Erlbaum. 
Pellegrino, J. W., \& KaIL, R. (1982). Process analyses of spatial aptitudes. In R. J. Sternberg (Ed.), Advances in the psychology of human intelligence (Vol. I). Hillsdale, NJ: Erlbaum.

Posner, M. I. (1978). Chronometric explorations of mind. Hillsdale, NJ: Erlbaum.

Posner, M. I., \& KeELE, S. W. (1968). On the genesis of abstract ideas. Journal of Experimental Psychology, 77, 353-363.

Robertson, L. C., \& PALMer, S. E. (1983). Holistic processes in the perception and transformation of disoriented figures. Journal of Experimental Psychology: Human Perception and Performance, 10, 231-249.

SHEPARD, R. N., COOPER, L. A. (1982). Mental images and their transformations. Cambridge, MA: MIT Press.

SHEPARD, R. N., \& METZLER, J. (1971). Mental rotation of three dimensional objects. Science, 171, 701-703.

SHINAR, D., \& OWEN, D. H. (1973). Effects of form rotation on the speed of classification: The development of shape constancy. Perception \& Psychophysics, 14, 149-154.

Simion, F., Bagnara, S., Roncato, S., \& Umiltà , C. (1982). Transformation processes upon the visual code. Perception \& Psychophysics, 31, 13-25.
Smith, K. U., Cambria, R., \& Stefan, J. (1964). Sensory-feedback analysis of reading. Journal of Applied Psychology, 48, 275-286.

WHITE, M. J. (1980). Naming and categorization of tilted alphanumeric characters do not require mental rotation. Bulletin of the Psychonomic Society, 15, 153-156.

Young, J. M., Palef, S. R., \& Logan, G. D. (1980). The role of mental rotation in letter processing by children and adults. Canadian Journal of Psychology, 34, 265.

\section{NOTE}

1. This second phase was added because an earlier pilot study had indicated that the transition to "normal"-" "reflected" decisions in the mental rotation task was difficult.

(Manuscript received June 15, 1984; revision accepted for publication March 28, 1985.) 